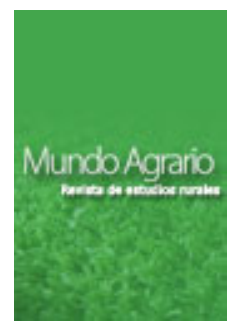

Mundo Agrario vol. 18, no 39, e072, diciembre 2017. ISSN 1515-5994

Universidad Nacional de La Plata.

Facultad de Humanidades y Ciencias de la Educación.

Centro de Historia Argentina y Americana

\title{
Estructura agraria y trabajo en un contexto de cambios: el caso de Uruguay 1
}

\section{Labour and agrarian structure in the context of changes: the case of Uruguay}

\section{Joaquín Cardeillac Gulla *, Agustín Juncal Pérez *}

* Universidad de la República - Departamento de Sociología - Núcleo de Estudios Sociales Agrarios, Uruguay| agustin.juncal@cienciassociales.edu.uy

\section{Palabras Clave}

Estructura agraria

Estructura social

Trabajadores rurales

Residencia

Uruguay

\section{KEYWORDS}

Agrarian structure

Social structure

Rural workers

Residency

Uruguay

\section{RESUMEN}

El artículo describe los cambios de la estructura agraria y la estructura social que ocurrieron en Uruguay entre 1980 y 2011. Mediante información censal, se muestra la disminución del número absoluto de asalariados agropecuarios, que se da junto al aumento de su importancia relativa frente a otros tipos sociales vinculados al sector, particularmente en relación a los productores familiares. Por otro lado, se observan modificaciones en las zonas de residencia de estos asalariados, que comienzan a cuestionar esa fuerte restricción a la elección del lugar de residencia, tradicional entre los trabajadores rurales.

\section{ABSTRACT}

The article describes the changes in the agrarian and social structure that occurred in Uruguay. Using census data from 1980 to 2011, the decrease in the absolute number of agricultural workers is shown, meanwhile it's relative participation in the economic active population grow, specially compared to family farmers. Besides, changes are observed in the areas of residence of these workers that question the strong restriction on the choice of residence place that had been traditional among rural workers. 


\section{Introducción}

En los últimos años, varios trabajos, aun con enfoques distintos, han coincidido en alertar acerca de los profundos cambios registrados en el agro uruguayo (Errea, Peyrou, Secco y Souto, 2011; Piñeiro y Moraes, 2008). En ese sentido, se ha mostrado cómo la penetración del capital financiero en el mercado de tierras generó un fuerte proceso de concentración y extranjerización del control de la misma (Piñeiro , 2010; Piñeiro , 2014). De modo concurrente, ha operado un cambio muy importante en las formas de organizar el negocio agropecuario, de suerte tal que en la actualidad han proliferado los arreglos de producción en red (Errea et al., 2011; Bisang, Anlló y Campi, 2008), que son expresiones locales de una tendencia global (Gereffi, Korzeniewicz \& Korzeniewicz, 1994).

En el marco de estos cambios en la propiedad de la tierra y en la forma en que se organiza el capital en el agro, el presente trabajo aborda una discusión sobre los impactos que estos fenómenos han tenido sobre el trabajo asalariado en el sector para el caso de Uruguay. Con ese objetivo, se analizan las principales tendencias que es posible establecer a partir de la información censal disponible para el período que va entre 1985 y 2011.

El artículo se divide en cinco apartados. El primero, repasa las principales transformaciones productivas del agro uruguayo. El segundo, presenta la situación de los asalariados agropecuarios dentro de la estructura social agraria. El tercero, expone los cambios en la distribución en el territorio de esos asalariados, mientras que el cuarto analiza los cambios observados en el tipo de ocupación de los mismos. Finalmente, un último apartado se destina a las conclusiones del estudio.

\section{Transformaciones productivas del agro uruguayo. Una mirada de largo plazo}

La cuestión agraria uruguaya presenta algunos rasgos singulares en comparación con el contexto latinoamericano debido a su temprana modernización agraria (1870-1914). De ese modo, se podrían enumerar los siguientes elementos distinguibles a fines del siglo XIX: a) la ausencia de población originaria vinculada a la tierra; b) un prematuro cierre de la frontera agrícola (Moraes, 2008); c) la conformación de un mercado de trabajo rural moderno (Moraes, 2003); d) la temprana inserción de bienes primarios en los mercados europeos; e) la instauración de un sistema de clases sustentando en relación con la posesión de medios de producción (tierra y capital) y la escala ocupacional (Errandonea, 1970), etc.

Entre 1870 y 1913, Uruguay presenció un importante crecimiento del producto ganadero a una tasa acumulativa próxima al 3\%, con un crecimiento de la productividad factorial total (PFT) en la ganadería que se situaba en el 2\% (Piñeiro y Moraes, 2008). La principal diferencia respecto a otros países, con los que se podría comparar el crecimiento agropecuario, radica en que al tiempo que Argentina, Canadá y Australia (por mencionar algunos ejemplos) aumentaron su producto a partir de la expansión de la frontera agrícola, Uruguay logró expandir su producto a partir del crecimiento de la productividad factorial total (PFT). El trabajo de Moraes (2008) constata que el crecimiento de los factores productivos se derivó de una trayectoria tecnológica que tuvo tres pilares: a) la incorporación del ovino a los establecimientos ganaderos; b) el establecimiento de los nuevos derechos de propiedad bajo la instauración del cercamiento de los campos; y c) la mestización del rodeo vacuno.

Los resultados más evidentes de este proceso se observan en la conformación de tres mercados diferentes que anteriormente no podían ser desligados entre sí y que confluían en la estancia ganadera. Con la modernización fue posible delimitar los mercados de tierras, ganados y trabajo (Moraes, 2008). Finalmente, un aspecto clave para entender este proceso fue que la transformación tecnológica, ocurrida durante el último cuarto de siglo XIX, surgió con innovaciones que se hicieron desde el latifundio ganadero, lo que acentuó aún más la relación dicotómica entre latifundio y minifundio (Moraes, 2008).

No obstante, el período de crecimiento durante la modernización (1870-1913) fue rápidamente sustituido por un desempeño económico agropecuario que se caracterizó por el estancamiento. En ese marco, el transcurso del siglo XX estuvo signado por la concepción de que la producción agropecuaria se había estancado desde la década de 1930. 
Las consecuencias del estancamiento ganadero son apreciables en varios acontecimientos económicos, sociales y políticos del país. Astori (1984) sostiene que el estancamiento ganadero podía apreciarse en tres aspectos. Primero, en el estancamiento de la producción a largo plazo dado que, durante las décadas de los treinta a los ochenta, se aprecia en el resto de América latina un crecimiento de la producción agropecuaria superior a Uruguay (con la única excepción de la pampa húmeda argentina). Allí hay tres aspectos de la realidad nacional que contrastaban con los demás países: a) el menor dinamismo tecnológico; b) el menor estímulo de la demanda de productos (tanto interna como externa); y c) una menor transnacionalización del capital asociado al agro uruguayo (predominio del capital nacional).

En segundo término, y siguiendo la línea interpretativa de Astori (1984), este proceso de estancamiento estuvo acompañado por cambios en la composición de la producción. De ese modo, el panorama uruguayo se caracterizaba por no poder expandir su frontera agrícola, y por no contar con posibilidades de cambios tecnológicos. Así, este autor sugiere que el escaso crecimiento observado se generó a partir de los cambios que se dieron en la estructura de la producción. Otros rubros agropecuarios no ganaderos fueron los que, en diferentes momentos del siglo XX, otorgaron dinamismo a la producción del sector (lechería, arroz, cultivos sacarígenos, etc.).

En tercer término, Astori (1984) planteaba que el estancamiento ganadero guardaba estrecho vínculo con cuatro aspectos que se podían identificar en la economía nacional: a) el deterioro tanto de las relaciones con el exterior como de la disponibilidad de reservas internacionales, lo que propició importantes desequilibrios financieros; b) el proceso interno de acumulación. Luego de la crisis de los treinta, el papel central de la ganadería (suplantada en los cuarenta y cincuenta por el rol estatal que impulsó la industrialización) no sería retomado nuevamente en los sesenta; c) acentuó el problema de abastecimiento de materias primas a la industria interna; d) por último, significó un severo problema para la retención de población rural por medio del empleo, por lo que el estancamiento fue un ingrediente más para el proceso de éxodo rural.

En resumen, en los años cincuenta el estancamiento ganadero ingresó en la agenda académica y política como un tema central para el desarrollo del país. Posteriormente, las décadas del sesenta y setenta estuvieron colmadas de diagnósticos y propuestas para superar el estancamiento ganadero.

No obstante, en las décadas de los setenta y ochenta, al igual que en los procesos registrados en el resto de América Latina, en Uruguay existe cierto consenso respecto a los fenómenos de intensificación capitalista. Uno de los factores clave que influyó en el proceso fue la promoción por parte del Estado de la diversificación productiva, como una forma de incentivar la exportación de rubros no tradicionales. A partir de 1970 se comenzaron a promocionar rubros tales como el arroz, el citrus, la cebada cervecera, la lechería y más tarde la forestación, generando fuertes inversiones desde sectores no agrícolas hacia los complejos agroindustriales (al igual que en bienes agrícolas, sistemas de investigación, transferencia tecnológica) con la finalidad de lograr mayor productividad del suelo y del trabajo. De esa manera, Piñeiro y Moraes (2008) sostienen que

hacia la mitad del siglo XX cobró fuerza entre los analistas del sector la noción de que la producción agraria estaba estancada, y tras varios estudios académicos, hacia 1970 se generalizó el concepto de "estancamiento dinámico" para aludir a una tendencia de muchas décadas al crecimiento casi nulo del producto agrario por habitante. El calificativo de “dinámico” expresaba la noción de que los dos grandes subsectores agrícola y ganadero presentaban comportamientos diferentes en materia de crecimiento, y también de que adentro de cada subsector se producían cambios en la composición tales que cuando un rubro -por ejemplo la carne- crecía, su contraparte -por ejemplo la lana- disminuía. (p. 10).

Desde comienzos del siglo XXI, se aprecia una serie de acontecimientos que revierten el proceso de estancamiento dinámico que caracterizó al agro uruguayo hasta ese momento (Piñeiro y Moraes, 2008). Esos acontecimientos permiten reactivar el crecimiento económico del sector agropecuario. Más en concreto, es habitual relacionar este proceso con el desarrollo de la cadena forestal y la agricultura de secano -específicamente, con la soja- aunque también con innovaciones en rubros tradicionales, como la ganadería y la lechería, donde 
empresas multinacionales dirigieron capitales. Más de diez años después del comienzo de estas modificaciones, las fuentes de información secundarias censales permiten evidenciar algunos resultados sobre la estructura agraria y social del agro uruguayo.

En cuanto a la estructura agraria, el último Censo General Agropecuario (CGA) de 2011, permite apreciar una notable modificación del paisaje agrario en ciertos territorios, debido a nuevos usos del suelo asociados a la forestación y los cultivos de cereales. Así, la forestación aumentó su área de 70.259 ha (1990) a 1.265.843 ha (2011) en tan solo 20 años. Algo similar ocurre con los cultivos de cereales y oleaginosas, pero en un período más acotado: aumentaron su superficie de 716.008 ha (2000) a 2.068.703 ha (2011) (Saavedra y Fagúndez, 2013). Por otro lado, la concentración de la tierra se aprecia en el pronunciado descenso de las explotaciones agropecuarias, que pasan de 57.131 a 44.890 entre 2000 y 2011 (Saavedra y Fagúndez, 2013). Ahora bien, lo que resulta más significativo del cambio en el número de explotaciones es que en todos los estratos (según tamaño de superficie) se observan disminuciones del número de explotaciones, a excepción del estrato de predios con más de 1000 ha de superficie. Por lo tanto, el período se caracteriza por un desplazamiento de tierras desde explotaciones "chicas" y "medianas" (conformadas por las que tienen entre 1 ha y 999 ha) hacia explotaciones "grandes" (de 1.000 ha y más). Otra tendencia que puede apreciarse, en el período referido, es la acentuación de un proceso de extranjerización de la tierra. El dato más revelador resulta del aumento de la participación de sociedades anónimas en el total de la superficie agropecuaria explotada del país, que llega a ser un $43 \%(2011)^{2}$. Si bien no es posible determinar cuáles son las nacionalidades que integran las diferentes sociedades anónimas, siguiendo a Piñeiro (2013) es posible afirmar que buena parte de las mismas corresponden a inversores extranjeros.

Todos los cambios reseñados están estrechamente vinculados con modificaciones en la estructura agraria y en la distribución de la tierra, y se hacen notorios al estudiar la evolución del número de explotaciones agropecuarias en los últimos años.

Gráfico 1. Cambio en el número de explotaciones agropecuarias (1980-2011)

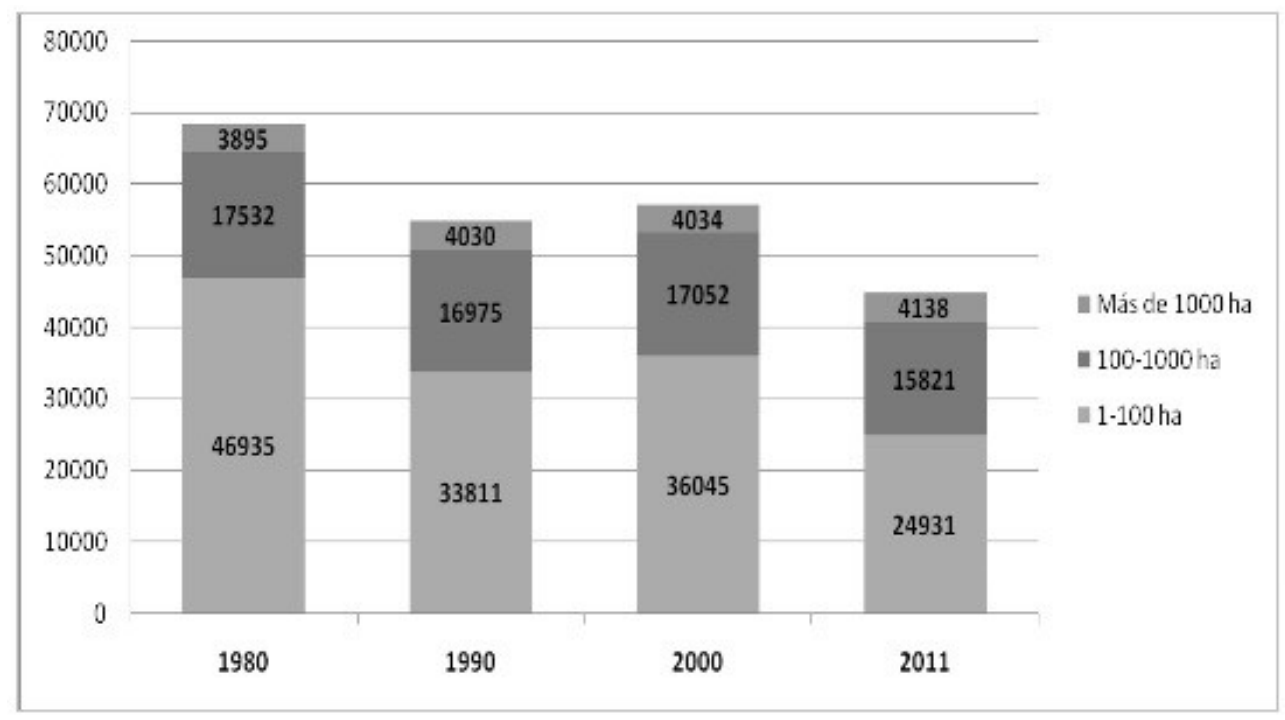

Fuente: elaborado en base a los Censos Generales Agropecuarios del MGAP

El Gráfico 1 permite apreciar las variaciones en el número de explotaciones agropecuarias registradas por los Censos Generales Agropecuarios (CGA), realizados por el Ministerio de Ganadería, Agricultura y Pesca (MGAP). A los efectos de la discusión que se presenta en este trabajo, resulta de interés retomar los resultados obtenidos entre 1980 y 2011. Del análisis resulta que se ha registrado un descenso muy marcado del número de explotaciones, primero entre 1980 y 1990 y luego entre 2000 y 2011. Adicionalmente, se destaca que el descenso en el número total está concentrado en las explotaciones de menor superficie, que generalmente corresponden a la producción familiar. Así, resulta que las explotaciones con superficies entre 1 hectárea y 100 hectáreas en 2011, son poco más que la mitad de las que había en 1980. En marcado contraste, para el caso de las explotaciones entre 
100 hectáreas y 1000 hectáreas la disminución del número fue de menos de un 10\%, y para el caso de las explotaciones con superficies mayores a 1000 hectáreas se da un aumento del 6\%. En el Gráfico 2 puede verse que estas tendencias, contrastantes en el número de explotaciones de los distintos estratos de tamaño, redunda en una modificación del peso relativo de los distintos tipos de explotación en cuanto al número de hectáreas acumuladas.

\section{Gráfico 2. Cambio en el número de hectáreas acumuladas por tamaño de la explotación (1990-2011) $\underline{3}$}

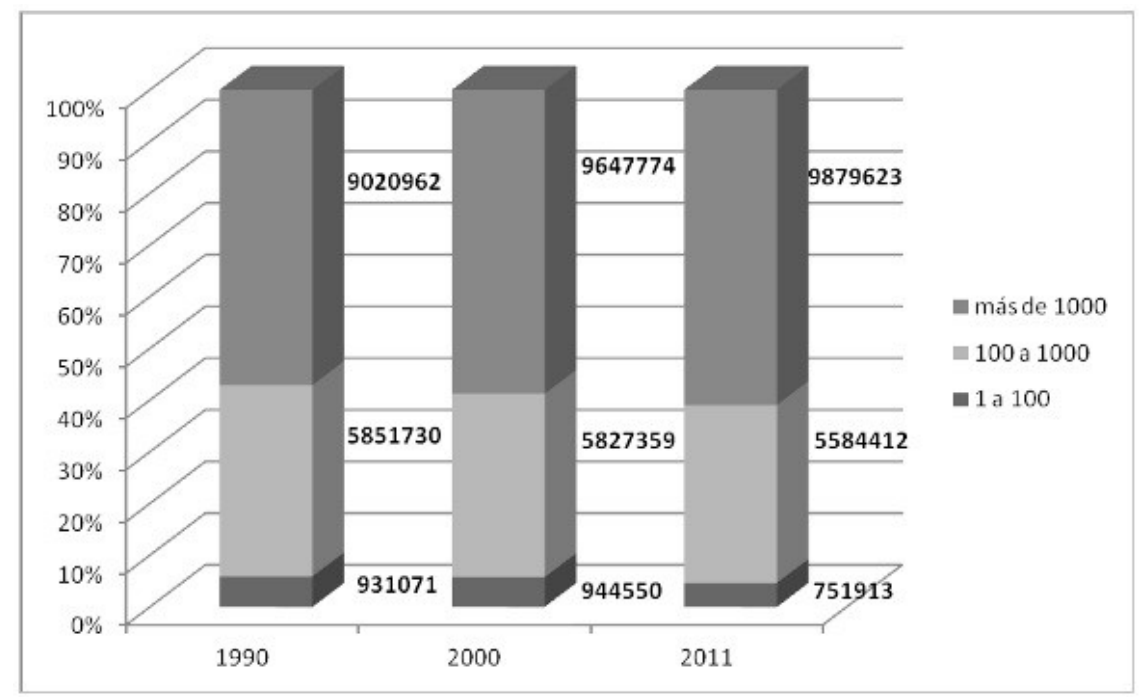

Fuente: elaborado con base en los CGA del MGAP.

El Gráfico 2 permite apreciar el cambio en la participación relativa de las explotaciones de distintos estratos de tamaño, en cuanto a las hectáreas que acumulan. El resultado más claro es que las explotaciones de mayor tamaño (más de 1000 ha) aumentan su participación relativa en la cantidad de hectáreas acumuladas, en más de 200.000 ha. Así, resulta que un aumento de poco más de 100 explotaciones entre 2000 y 2011 (Gráfico 1) es suficiente para lograr un aumento de más de 200.000 ha en superficie (Gráfico 2), lo cual es un indicio adicional del proceso de concentración de la tierra referido por los antecedentes.

En el próximo apartado se presentarán los resultados que se obtienen al analizar los cambios en la estructura social que se dan en este mismo período.

\section{Asalariados agropecuarios y estructura social agraria}

Para estudiar los cambios en la estructura social del Uruguay se ha optado por trabajar en base a los Censos Nacionales de Población y Vivienda (CNPV) y no en base a los GCA. Esta opción se realiza en atención al hecho de que los CGA tienen un claro sesgo a relevar variables asociadas a la producción y no a aspectos laborales o sociales. Adicionalmente, las unidades de análisis de las dos fuentes de datos son distintas. En el caso de los Censos Agropecuarios las unidades de análisis son las explotaciones de al menos 1 ha, mientras que en los CNPV son las personas (también hogares y viviendas). Si bien el manejo de fuentes distintas puede complejizar el análisis, a los efectos de la argumentación que aquí se viene realizando la utilización de fuentes diferentes robustece al argumento, en la medida en que resultados obtenidos de ambas fuentes validan por concurrencia el sentido de los cambios que se proponen para el caso de la estructura social y agraria del Uruguay rural.

A la luz de las modificaciones de la estructura agraria y la distribución de la tierra recién presentadas, resulta pertinente avanzar en una contextualización que permita aproximar algunos indicios de los cambios que se dan en la estructura social del Uruguay. Al respecto, los datos disponibles para el período que va entre 1985 y 2011 evidencian los efectos de los grandes cambios que han ocurrido en el medio rural uruguayo. 
Cuadro 1: Cambios en la estructura social agropecuaria (1985-2011)

\begin{tabular}{|l|c|c|c|c|c|c|}
\hline & $\mathbf{1 9 8 5}$ & $\begin{array}{c}\text { Participación } \\
\text { relativa \% }\end{array}$ & $\mathbf{1 9 9 6}$ & $\begin{array}{c}\text { Participación } \\
\text { Relativa \% }\end{array}$ & $\begin{array}{c}\mathbf{2 0 1 1} \\
\begin{array}{c}\text { Participación } \\
\text { Relativa \% }\end{array}\end{array}$ \\
\hline Asalariado privado & 92.126 & 56 & 69.649 & 52 & 69.284 & 65 \\
\hline $\begin{array}{l}\text { Trabajador por cuenta } \\
\text { propia }\end{array}$ & 43.685 & 27 & 33.494 & 25 & 19.219 & 18 \\
\hline $\begin{array}{l}\text { Trabajador familiar no } \\
\text { remunerado }\end{array}$ & 12.458 & 8 & 11.830 & 9 & 4.595 & 4 \\
\hline Patrón & 14.561 & 9 & 18.765 & 14 & 12.577 & 12 \\
\hline $\begin{array}{l}\text { Miembro de cooperativa de } \\
\text { producción }\end{array}$ & 591 & 0,1 & 622 & 0,1 & 161 & 0,1 \\
\hline Total & 163.421 & 100 & 134.360 & 100 & 105.836 & 100 \\
\hline
\end{tabular}

Fuente: elaboración propia en base a los datos de los CNPV del INE de 1985, 1996 y 2011.

El Cuadro 1 presenta el número absoluto de ocupados de la rama 1 (Producción agropecuaria, forestación y pesca), según las categorías de ocupación construidas en base a las relaciones con los medios de producción (Riella, Florit y Vitelli, 2011), para cada uno de los años en que se realizó un censo de población entre 1985 y 2011. Adicionalmente, se presenta la participación relativa de cada una de esas categorías para cada año, como forma de evidenciar más claramente el cambio en el peso de cada uno de estos colectivos dentro del total de ocupados en el sector.

A partir del análisis resulta posible observar dos períodos bien distintos. Por un lado, un período (1985-1996) en el que el número absoluto de personas vinculadas al sector primario de la economía disminuye, al tiempo que su composición por categoría de ocupación se mantiene relativamente estable, lo cual ha sido asociado a la temprana introducción de relaciones capitalistas de producción en el agro uruguayo (Riella et al., 2011). Por otro lado, se observa un período de fuertes modificaciones, el que va entre 1996 y 2011.

Así, en este segundo período, el número de personas vinculadas al sector primario vuelve a disminuir, pero con la diferencia de que las reducciones no se dan con la misma intensidad en todas las categorías de ocupación.

El Gráfico 3, que resume la información de la tabla anterior agrupando las categorías Cuenta Propia y Trabajadores Familiares, permite observar más claramente las diferencias de los períodos en relación a los cambios en la composición por clase de los ocupados en el sector.

\section{Gráfico 3. Cambios en la estructura social agraria (1985-2011)}

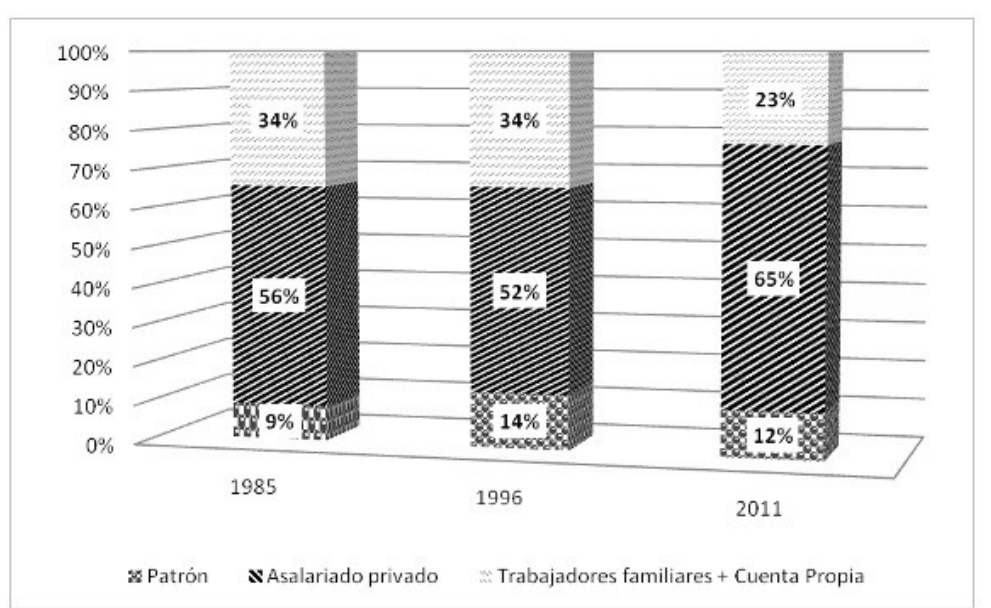

Fuente: elaboración propia en base a los datos de los CNPV del INE de los años 1985, 1996 y 2011. 
En concreto, se observa que los asalariados agropecuarios pasan de representar un 52\% en 1996, a un 65\% de los ocupados en el sector primario en el 2011. Por su parte, las categorías de cuenta propia y trabajadores familiares, que suelen utilizarse como forma de aproximar a los productores familiares (Piñeiro y Cardeillac, 2014; Riella , 2010), pasan de acumular un 34\% de los ocupados del sector a sólo un 23\% en 2011, y también el número de ocupados que se declaran patrones disminuye su participación relativa, aunque sólo dos puntos porcentuales, de $14 \%$ en 1996 a un $12 \%$ en 2011.

Llegado este punto, se han mostrado algunas tendencias relativas a los cambios en la distribución de la tierra en el campo uruguayo, así como su correlato en términos de modificaciones en las categorías de ocupación de la población vinculada económicamente con el sector agropecuario. Al respecto, se pueden identificar dos momentos en los que se dan cambios de gran magnitud en relación con la distribución de la tierra y que ambientan, a su vez, modificaciones de distinto tipo en términos de la población.

El primer momento de cambios se da entre 1980 y 1990. Allí se observa una disminución muy importante del número de explotaciones agropecuarias, y en particular de aquellas más pequeñas, de entre 1 hectárea y 100 hectáreas. Ahora bien, en términos de la población económicamente vinculada al sector agropecuario, los cambios que se observan pasan por una disminución del total de población económicamente vinculada con el sector agropecuario de un $18 \%$, que se modifica entre las distintas categorías de ocupación o estratos sociales. Así, mientras el número de explotaciones de menos de 100 hectáreas se reduce en un $28 \%$ en 10 años, el porcentaje correspondiente a población asalariada se reduce en un $28 \%$ (es decir, un $10 \%$ más que el total de población), el número de trabajadores familiares o cuenta propia cae en un 23\% y el número de patrones aumenta un $29 \%$.

En consecuencia, puede afirmarse que el proceso de concentración de la tierra que se da entre 1980 y 1990 tuvo, como consecuencia fundamental, una disminución del número de personas trabajadoras asalariadas directamente vinculadas al sector primario, así como de las personas vinculadas a la producción familiar.

El segundo momento de cambios corresponde al período que va entre 2000 y 2011. En este período se vuelve a observar una marcada disminución del número de explotaciones más pequeñas, que se reducen nuevamente un $30 \%$. No obstante, al analizar lo sucedido con la población económicamente vinculada al sector -que en este contexto funciona como indicador de la estructura social rural-, los cambios son radicalmente distintos. Así, si bien entre 1996 y 2011 (años para los que se dispone de información Censal) la población vinculada con el sector vuelve a caer en el entorno de un 20\%, las caídas según categorías de ocupación son muy diferentes.

Por un lado, el número absoluto de trabajadores asalariados casi no varía, mientras que, por otro lado, la población vinculada a la producción familiar cae dramáticamente. Una consecuencia de estos dos movimientos es el marcado aumento del peso relativo de los trabajadores asalariados agropecuarios, que alcanzan a ser en 2011 un 65\% de la población vinculada laboralmente al sector (Gráfico 3).

Sintetizando lo analizado hasta el momento, los resultados obtenidos muestran que el segundo período de concentración de la tierra revisado ha tenido un impacto muy distinto al observado entre 1980 y 1990 . A diferencia de lo que aconteció entonces, los cambios experimentados entre 2000 y 2011 no han redundado en una desaparición de trabajadores asalariados. Por el contrario, el proceso de concentración de tierras de inicios del siglo XXI se ha dado al mismo tiempo que se modifican las formas de organizar el trabajo en la producción agropecuaria, y el resultado de ambas tendencias es la merma de una forma específica de organizar la producción agropecuaria: la producción que se basa en trabajo familiar. Al mismo tiempo, la producción basada en trabajo asalariado aumenta su participación relativa, acompañando el aumento de la concentración de la tierra en relativamente pocas empresas de mayor tamaño.

Tomando como contexto estos cambios estructurales recién esbozados, en adelante se avanzará en el estudio de las características de los trabajadores asalariados vinculados al sector primario en Uruguay. 


\section{Cambios en la distribución en el territorio de los asalariados agropecuarios}

Si bien el aspecto central que define a un trabajador como asalariado rural tiene que ver con el tipo de tareas que realiza (Juncal, Cardeillac, Moreira y Gallo, 2014; Carámbula, 2009; Piñeiro , 2008), es un hecho que, para el sentido común, la realidad de los asalariados agropecuarios se asocia a la vida rural en un sentido bien específico: la residencia fuera de localidades nucleadas.

Así, aun cuando existe una vasta producción académica que discute esa idea y muestra la variedad de formas en las que el trabajo rural se puede articular con distintos lugares de residencia (Piñeiro y Cardeillac, 2014; Cardeillac, 2013; Riella y Mascheroni, 2009; Piñeiro , 2001), resulta interesante analizar cómo y en qué magnitud han habido cambios de las zonas donde habitan más frecuentemente los asalariados agropecuarios.

El Cuadro 2 presenta la distribución de los asalariados del sector primario para los tres años en que se dispone de información censal, según si residían en localidades de hasta 2000 personas, en localidades de entre 2000 y 5000 personas, en localidades de más de 5000 personas, o bien como población dispersa, es decir, como población que reside fuera de zonas amanzanadas o localidades, lo que según el Instituto Nacional de Estadística (INE) es considerado “población rural”.

Cuadro 2: Cambios en el lugar de residencia de los asalariados agropecuarios (1985-2011)

\begin{tabular}{|l|c|c|c|c|c|c|}
\hline & \multicolumn{2}{|c|}{1985} & \multicolumn{2}{c|}{1996} & \multicolumn{2}{c|}{2011} \\
\hline Población dispersa & 65.495 & $69 \%$ & 42.281 & $61 \%$ & 21.020 & $30 \%$ \\
\hline $\begin{array}{l}\text { Localidades de menos de 2000 } \\
\text { personas }\end{array}$ & 8.585 & $9 \%$ & 7.027 & $10 \%$ & 12.304 & $18 \%$ \\
\hline $\begin{array}{l}\text { Localidades de más de 2000 y } \\
\text { menos de 5000 personas }\end{array}$ & 3.493 & $4 \%$ & 3.708 & $5 \%$ & 5.886 & $8 \%$ \\
\hline $\begin{array}{l}\text { Localidades de más de 5000 } \\
\text { personas }\end{array}$ & 17.094 & $18 \%$ & 16.633 & $24 \%$ & 30.074 & $43 \%$ \\
\hline Total & 94.667 & $100 \%$ & 69.649 & $100 \%$ & 69.284 & $100 \%$ \\
\hline
\end{tabular}

Fuente: elaboración propia en base a los CNPV del INE de 1985, 1996 y 2011.

A partir del análisis vuelve a ser posible la identificación de tendencias distintas por período. De hecho, aunque en el caso de los cambios en la zona de residencia ya en el período 1985-1996 era posible observar una tendencia a dejar de residir como población dispersa, resulta evidente que la parte más importante de los cambios se da entre 1996 y 2011.

Analizando todo el período, se observa que entre 1985-1996 y 2011 las localidades de menos de 2000 personas pasan de acumular entre un 4\% (1985) y un 5\% (1996) de los asalariados agropecuarios, a acumular un 8\% (2011), mientras que las localidades de entre 2000 y 5000 personas pasan de entre un 9\% y un 10\% a un 18\% para los mismos años. Dicho de otro modo, las localidades de menos de 5000 habitantes duplicaron su participación relativa entre 1985 y 2011, y esto es así básicamente por lo ocurrido entre 1996 y 2011.

A su vez, las localidades de mayor tamaño del país, es decir, las de más de 5.000 habitantes, pasaron de acumular entre un 18\% y un 24\% según el año, a concentrar un 43\% de los asalariados agropecuarios en 2011, lo cual implica un crecimiento aun mayor que el de las ciudades y pueblos más pequeños. Por último, y como contracara del crecimiento de la proporción de asalariados que residen en localidades nucleadas, se observa que los asalariados agropecuarios que residen como población dispersa pasan de ser entre siete y seis de cada diez en 1985-1996, a ser apenas tres de cada diez en 2011. 


\section{Análisis del tipo de ocupación de los asalariados agropecuarios en 1996 y 2011}

Varios estudios sobre los trabajadores agropecuarios han manejado definiciones más o menos diferentes (Cardeillac, Gallo y Moreira, 2013; Buxedas, Perera y Barrios, 2012; Mascheroni, 2011), que redundan necesariamente en resultados distintos. Para el caso de la información que se presenta de aquí en adelante, se retomarán las reflexiones y recomendaciones realizadas en el trabajo de Juncal et al. (2014). Los autores, retomando aspectos discutidos por Latorre (1993), Errandonea (1987) y Guerra Daneri (1983) plantean, para el estudio de las condiciones de vida de los asalariados agropecuarios, la necesidad de distinguir entre los diferentes tipos de ocupaciones que se pueden observar al interior de los diferentes rubros de producción y que están vinculados al sector agropecuario. En tal sentido, la información que se presenta en el Cuadro 3 corresponde al subconjunto de ocupados asalariados privados de la rama 1, que se desempeñan en la agropecuaria o forestación, dejando fuera del análisis a los trabajadores de la pesca.

\section{Cuadro 3: Tipos de ocupaciones de los asalariados de la actividad agropecuaria y forestal}

\begin{tabular}{|l|c|c|c|c|}
\hline \multirow{2}{*}{ Administradores, profesionales y técnicos } & \multicolumn{2}{|c|}{1996} & \multicolumn{2}{c|}{2011} \\
\cline { 2 - 5 } & Número & \% & Número & 2,9 \\
\hline Personal de oficina y servicios & 766 & 1,1 & 2.006 & 8,2 \\
\hline Trabajadores agropecuarios calificados & 2.682 & 3,9 & 5.567 & 24,9 \\
\hline Operadores de vehículos y maquinaria & 19.063 & 27,7 & 16.989 & 10,7 \\
\hline Trabajadores agropecuarios no calificados & 5.680 & 8,3 & 7.273 & 36,0 \\
\hline Otras ocupaciones no calificadas & 35.122 & 51,1 & 24.540 & 10,0 \\
\hline Ignorado & 4.674 & 6,8 & 6.851 & 7,3 \\
\hline Total & 709 & 1,0 & 4.974 & 100 \\
\hline
\end{tabular}

Fuente: elaboración propia en base a los CNPV del INE de 1985, 1996 y 2011.

Partiendo de estos datos es posible observar cambios adicionales entre los años 1996 y 2011. Si bien el número total de trabajadores agropecuarios no se altera mucho, sí se registran modificaciones a la interna entre los distintos tipos de ocupaciones. Para facilitar la interpretación, en la tabla se han agrupado distintas categorías de ocupación que distinguen los clasificadores de ocupaciones que utilizó el INE en cada año ${ }^{4}$.

En cuanto a los cambios, la información permite apreciar que el número de asalariados calificados con tareas agropecuarias no se reduce mucho (menos de 3 puntos porcentuales). En cambio, sí se observa una reducción significativa del peso de los asalariados no calificados que realizan tareas agropecuarias o forestales. De hecho, hacia 1996 más de la mitad de los asalariados agropecuarios $(51,1 \%)$ eran trabajadores no calificados, mientras que en 2011 sólo un poco más de un tercio (36\%) de los asalariados agropecuarios son clasificados así. Al mismo tiempo, se observa un fuerte aumento en términos relativos de la participación de otros tipos de ocupaciones entre los asalariados. Tanto los Administradores, profesionales y técnicos como el Personal de oficina y servicios, duplican su participación relativa en el conjunto de los asalariados agropecuarios, al tiempo que también aumentan los trabajadores no calificados con tareas no agropecuarias y también los operadores de vehículos y maquinaria.

El Gráfico 4 permite observar mejor los cambios entre 1996 y 2011, al omitir del total los casos en los que no se dispone de información. 


\section{Gráfico 4. Cambios en la participación relativa de los distintos tipos de ocupaciones entre los asalariados rurales (1996-2011)}

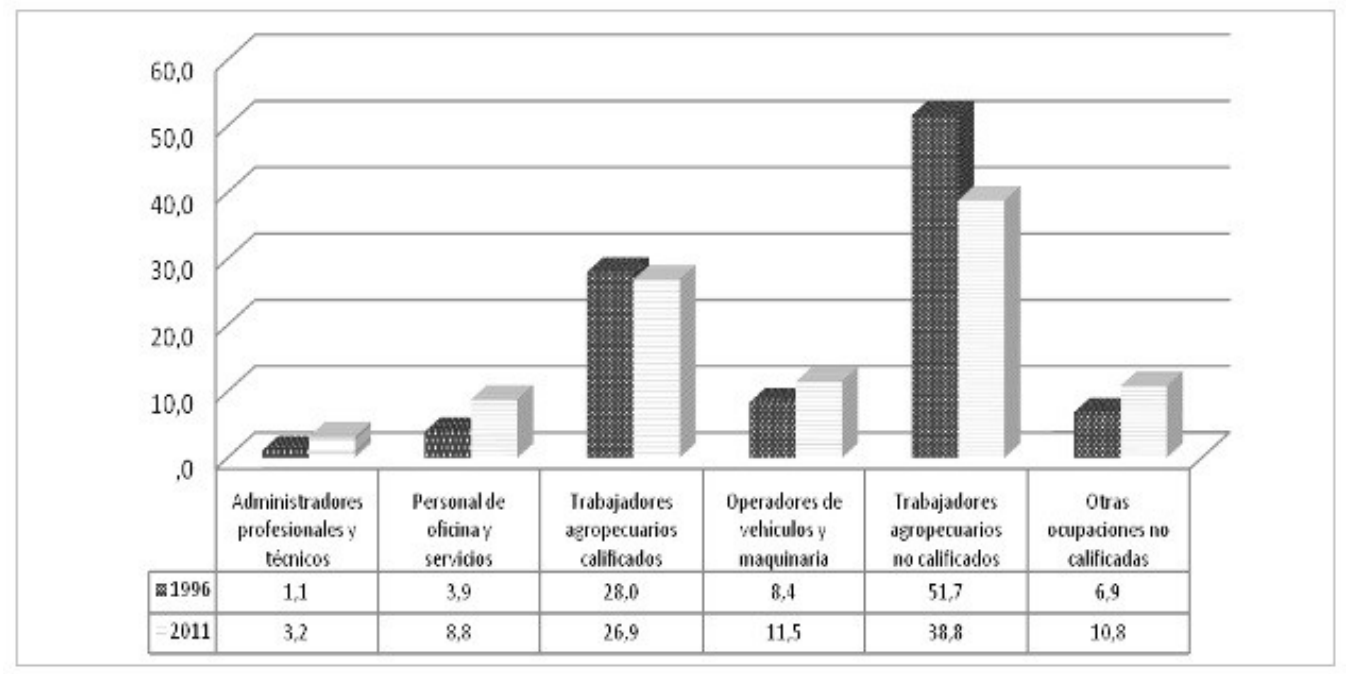

Fuente elaboración propia en base a los CNPV de 1996 y 2011 del INE.

Los resultados alertan sobre la necesidad de tener presentes los cambios en la composición interna del conjunto de los asalariados del sector agropecuario entre 1996 y 2011, ya que a los distintos tipos de ocupaciones presentadas suelen estar asociadas diferentes condiciones de empleo y de trabajo, así como diferentes posibilidades de acceso a ingresos y derechos (Juncal et al., 2014).

Más en concreto, el período que va de 1996 a 2011 se ha caracterizado por una modificación de la composición interna del tipo de ocupaciones que desarrolla la mano de obra asalariada vinculada al sector agropecuario y forestal. Así, lo que era la realidad de la mayoría absoluta de los asalariados rurales en 1996, estar ocupado en tareas agropecuarias no calificadas, es en 2011 una realidad que solo alcanza a un poco más de un tercio de los asalariados rurales. Al mismo tiempo, las ocupaciones no vinculadas a tareas agropecuarias han aumentado entre los asalariados rurales, tanto las no calificadas, como también las ocupaciones que exigen mayores calificaciones.

\section{Reflexiones finales}

Este artículo complementa varios trabajos y estudios previos (Carámbula et al., 2012; Cardeillac et al., 2013; Juncal et al., 2014) que permiten afirmar la existencia de brechas en el ejercicio de derechos por parte de los trabajadores rurales respecto al resto de los asalariados privados del Uruguay. Su propósito ha sido exponer las modificaciones de la estructura social agraria uruguaya a partir de los cambios productivos ocurridos en los últimos años. En tal sentido, el estudio permite establecer cinco puntos a modo de conclusiones.

En primer lugar, la interpretación de los resultados permite corroborar la idea de un agro uruguayo en el que las relaciones capitalistas de producción han ganado terreno, desplazando otras formas de producir (básicamente la producción de tipo familiar) que hasta hace no mucho tiempo habían mostrado una cierta capacidad de permanencia (Riella et al., 2011).

En segundo lugar, mientras las formas de producción que dependen de relaciones no asalariadas de producción pierden peso relativo, los ocupados que participan de relaciones asalariadas de producción pasan a representar casi dos de cada tres ocupados en el sector primario.

En tercer lugar, el perfil de los asalariados rurales también se ha visto modificado sustancialmente en el período 1996-2011. La residencia es primordialmente urbana (en buena parte en localidades de más de 5000 habitantes) y se registran cambios notorios en el tipo de tareas que realizan. 
En cuarto lugar, el análisis realizado de los cambios en la estructura social rural justifica los esfuerzos por profundizar en el análisis del colectivo de los asalariados rurales, ya que es el colectivo que más ha aumentado su participación relativa en el agro uruguayo contemporáneo, al mismo tiempo que se ha modificado en su composición y características.

Por último, el aumento del peso del trabajo asalariado, concomitante a la disminución del peso relativo de la producción familiar (tanto como forma de trabajo como en términos de la cantidad de tierra que acumula) y al aumento de escala de las explotaciones capitalistas, evidencian que el agro uruguayo atravesó un intenso ajuste entre el 2000 y el 2011. Cuánto más se profundizarán estas tendencias es una pregunta abierta. Por lo pronto, recorrer el camino hacia el reconocimiento de derechos asociados al trabajo entre los asalariados rurales y los esfuerzos de la política pública orientados a garantizar su efectivo goce, siendo siempre necesarios, resultan particularmente oportunos.

\section{Notas}

1 Este trabajo fue generado en el marco de la investigación: "Los límites de la Ciudadanía: el caso de los trabajadores asalariados rurales”, que financió la Comisión Sectorial de Investigación Científica (CSIC) de la Universidad de la República (UdelaR). Equipo: Joaquín Cardeillac, Diego Piñeiro; Matías Carámbula; Alejandra Gallo; Agustín Juncal; Bolivar Moreira. El proyecto buscó comprender los mecanismos sociales que operan propiciando u obstaculizando la efectiva apropiación de derechos por parte de estos asalariados, profundizando en aquellos que constituyen un factor clave para una eventual reducción de desigualdades en términos de bienestar. La investigación fue realizada entre 2013 y 2015.

$\underline{2}$ En el año 2000, todas las personas jurídicas con contrato (SRL, SA, etc.) no llegaban a acumular un 28\% del total de la superficie.

$\underline{3}$ Los números absolutos al costado de las barras corresponden al número de hectáreas acumulado por las explotaciones de cada tipo.

4 En el caso del Censo de 1996 se utilizó el CNUO95 (manual guía para la codificación de ocupaciones de actividad) Clasificación Internacional Uniforme de Ocupaciones (CIUO-88) Adaptada a Uruguay. En el caso del Censo de 2011 se utilizó el listado nacional de denominaciones de ocupaciones. Ambos disponibles en: http://www.ine.gub.uy/codificadores y simbologia/codificadores.asp

\section{Fuentes}

Dirección de Estadísticas Agropecuarias (DIEA). Ministerio de Ganadería, Agricultura y Pesca (MGAP). (2000). Censo General Agropecuario 2000 (archivo informático). (Área Socio Demográfica del Banco de Datos) Montevideo,Montevideo, Uruguay.

Dirección de Censos y Encuestas del MGAP. Ministerio de Ganadería, Agricultura y Pesca (MGAP). (1990). Censo General Agropecuario, 1990 (archivo informático). (Área Socio Demográfica del Banco de Datos) Montevideo, Montevideo, Uruguay.

Instituto Nacional de Estadística (INE). (2011). Censos 2011. Recuperado el 14 de 05 de 2016, de http://www.ine.gub.uy/censos2011/index.html

Instituto Nacional de Estadística (INE). (1996). VII Censo de población, III hogares y V de viviendas (archivo informático). Montevideo, Montevideo, Uruguay. 
Instituto Nacional de Estadística (INE). (1985). VI Censo de población, II hogares y IV de viviendas (archivo informático). Montevideo, Montevideo, Uruguay.

\section{Bibliografía}

Astori, D. (1984). Principales interpretaciones sobre la problemática agraria uruguaya. En La cuestión agraria en el Uruguay (pp. 39-68). Montevideo: FCU/CIEDUR.

Bisang, R., Anlló y G. Campi, M. (2008). Una revolución (no tan) silenciosa. Claves para repensar el agro en Argentina. Revista de Desarrollo Económico, 48, 189-190. Buenos Aires: IDES.

Buxedas, M., Perera, M. y Barrios, M. (2012). Caso de Uruguay. En Fernando Soto Baquero y Emilio Klein (Ed), Políticas de mercado de trabajo y pobreza rural en América Latina. Tomo II. (pp. 290-334). Santiago de Chile: Oficina Regional de la FAO para América Latina y el Caribe.

Carámbula, M. (2009). Tiempos de Ausencia. Movilidad espacial y precariedad laboral en los trabajadores rurales temporales: el caso de los esquiladores de Villa Sara. Montevideo: CSIC, Udelar. Letraeñe Ediciones.

Carámbula, M, Cardeillac, J., Gallo, A., Juncal, A., Moreira, B. y Piñeiro, D. (2012). Los límites de ciudadanía: El caso de los trabajadores asalariados rurales. En El Uruguay desde la Sociología X (pp. 351-370). Montevideo: Departamento de Sociología, Mastergraf S.R.L.

Cardeillac, J., Gallo, A. y Moreira, B. (2013). Entre el reconocimiento y la apropiación. Un análisis de las condiciones de vida de los asalariados rurales del Uruguay en un contexto de crecimiento económico y desarrollo social. Ponencia presentada en el Congreso de Sociología del Trabajo de ALAST, São Paulo.

Cardeillac, J. y Juncal, A., (2014). Políticas Públicas de Juventud dirigidas a jóvenes rurales. En INJU Plan de Acción de Juventudes 2015-2025: Estudios(pp. 113-129). Montevideo: Imprimex.

Errandonea, A. (1970). Apuntes sobre la conformación de las clases sociales en el medio rural uruguayo. En Cuadernos de Ciencias Sociales (pp. 9-54). Montevideo: Facultad de Derecho, Instituto de Ciencias Sociales.

Errandonea, A. (1987). Las clases sociales en el Uruguay. Centro Latinoamericano de Economía Humana (CLAEH): Ediciones de la Banda Oriental.

Errea, E., Peyrou, J., Secco, J., y Souto, G. (2011). Transformaciones en el agro uruguayo: Nuevas instituciones y modelos de organización empresarial. Montevideo: Universidad Católica del Uruguay.

Gereffi, G., Korzeniewicz, M. \& Korzeniewicz, R. (1994). Introduction: Global Commodity Chains. En Gereffi, G. \& Korzeniewicz, M. Commodity chains and global capitalism. USA: Praeger Publishers.

Guerra Daneri, E. (1983). Régimen jurídico del trabajador agrario. Montevideo: MZ Editor. Montevideo.

Juncal, A., Cardeillac, J., Moreira, B. y Gallo, A. (2014). Conceptualización de asalariados agropecuarios y caracterización de sus condiciones de vida en un contexto de crecimiento económico y desarrollo social del Uruguay.. En El Uruguay desde la Sociología 12 (pp. 259-271). Montevideo: Departamento de Sociología, Mastergraf S.R.L.

Latorre, R. (1993). Los trabajadores rurales en Uruguay. Montevideo: Facultad de Agronomía.

Mascheroni, P. (2011). Democracia y ciudadanía en el campo: los primeros Consejos de Salarios Rurales en Uruguay. Montevideo: UR-FCS-DS.

Moraes, M. (2003). El trabajo de la esquila y los esquiladores: algunos aspectos de su historia social (1860-1979). En Piñeiro, D. , Trabajadores de la esquila. Pasado y presente de un oficio rural (pp. 31-58). Montevideo: CSIC, Udelar. 
Moraes, M. (2008). La pradera perdida. Historia y economía del agro uruguayo: una visión de largo plazo 17601970. Montevideo: Librería Linardi y Risso. CSIC, Udelar.

Piñeiro, D. (2001). Población y trabajadores rurales en el contexto de transformaciones agrarias. En Giarracca, N. ¿Una nueva ruralidad en América Latina? (pp. 269-288).Buenos Aires: CLACSO. Recuperado de http://biblioteca.clacso.edu.ar/clacso/gt/20100929020330/13pineiro.pdf

Piñeiro, D. (2008). El trabajo precario en el campo uruguayo. Montevideo: Facultad de Ciencias Sociales. CSIC, Udelar.

Piñeiro, D. (2010). Concentración y extranjerización de la tierra en el Uruguay. En Las agriculturas familiares del MERCOSUR. Trayectorias, amenazas y desafíos (pp. 153-170). Buenos Aires: CICCUS

Piñeiro, D. y Cardeillac, J. (2014). Producción familiar y agronegocios: dos modelos en conflicto. Revista ALASRU. Asociación Latinoamericana de Sociología Rural, 10, 187-205.

Piñeiro, D. y Moraes, M. (2008). Los cambios en la sociedad rural durante el siglo XX. En Nahum, B. (Ed), El Uruguay del siglo XX. La sociedad. Montevideo: Ediciones de la Banda Oriental.

Riella, A., Florit, P. y Vitelli, R. (2011). Estructura de clases y desigualdad en el Uruguay Rural contemporáneo. En Espacio abierto. Cuaderno Venezolano de Sociología, 20(4), 37-80.

Riella, A. y Mascheroni, P. (2009). Población, ingresos y hogares agrodependientes. En Anuario OPYPA 2009 (pp. 461-468). Montevideo: MGAP.

Riella, A., Tubío, M. y Lombardo, R. (2013). Cadenas Globales y trabajo rural. Revista de Ciencias Sociales, 32(26), 113-132.

Saavedra, C. y Fagúndez, D. (2013). El Censo General Agropecuario 2011 y la nueva realidad agropecuaria. Revista del Plan Agropecuario, 145, 64-70. 